\title{
DIAGNÓSTICO DA METODOLOGIA BIM ENTRE OS ANOS DE 2017 E 2019 NO CENTRO DE TECNOLOGIA DA UFC
}

Sabrina R. Gonçalves - sabrinarg57@gmail.com

Camily V. Barbosa-vasconcelosmily@alu.ufc.br

Luciano H. C. H. Sousa-lucianohamed@alu.ufc.br

Antonio P.H. Cavalcante - apaulo@det.ufc.br

Departamento de Integração Acadêmica e Tecnológica - DIATEC

UFC. Campus do Pici, Bloco 710, 1o Andar

CEP 60455760 - Fortaleza - Ceará

\section{Resumo:}

A metodologia BIM vem ganhando cada vez mais importância no Brasil e a estratégia BIM BR corrobora isso. Nesse sentido, objetivamos realizar um diagnóstico sobre a percepção da metodologia BIM no contexto da Universidade Federal do Ceará, entre os anos de 2017 e 2019. Para tanto, foi realizada em 2017 uma pesquisa aos alunos do centro de tecnologia da UFC, onde foram questionados sobre a percepção da filosofia BIM no curso, a sua relevância e o interesse pela disciplina focada em BIM. Em 2020, foi realizada uma nova pesquisa com os estudantes para fins comparativos dos dados. Tangente a isso, foi realizado um levantamento de ações realizadas neste período e classificadas nas categorias: produção científica, eventos e capacitações. Dentre as categorias foi possível perceber que no ano de 2017 houve o maior número de TCC's que tratavam da metodologia, fato que coincidiu com o retorno de diversos alunos do exterior. Por outro lado, a quantidade de eventos relacionados organizados na universidade foram crescentes, demonstrando a relevância sobre a temática, dentre eles, temos eventos nacionais e internacionais. E ainda o número de capacitações realizado na universidade também se mostrou crescente, o que corrobora a importância da metodologia. Diante disso, no cenário onde temos a Estratégia BIM BR como um forte incentivo para o uso da metodologia, podemos inferir que apesar da produção científica relacionada à temática ter diminuído, as outras categorias crescentes mostram que a preocupação maior entre os discentes é o preparo para o mercado.

Palavras-chave: Diagnóstico. BIM. UFC.

\section{INTRODUÇÃO}

Apesar de ter ganhado mais força no século XXI, o conceito de Modelagem de Informação da Construção não é tão recente. Em 1974, Charles Eastman já falava sobre a importância de se ter um sistema de descrição da construção. G.A. van Nederveen e F.P. Tolman utilizaram pela primeira vez o termo BIM - Building Information Modelling - em 1992, em um estudo que ressalta a importância da modelagem da informação para um modelo de construção.

Dessa forma, segundo Eastman em seu livro Manual de BIM, com esta tecnologia, um modelo virtual preciso de uma edificação é construído de forma digital e este contém a geometria exata e os dados relevantes, necessários para dar suporte à construção, à fabricação e ao fornecimento de insumos necessários para a realização da construção. 
A definição de Kymell (2008) é mais abrangente ao percorrer o ciclo de vida do empreendimento como um todo. Para ele, BIM é a simulação de um projeto, que consiste na modelagem 3D dos componentes do mesmo, agregando-se toda a informação requerida ao seu planejamento, à construção ou operação, e ao encerramento do ciclo de vida da edificação.

Em suma, a modelagem da informação da construção reúne todas as informações necessárias para se planejar, executar e gerenciar uma construção, de forma que facilita a previsão de possíveis problemas na execução, minimizando custos e otimizando tempo. Para Addor (2015), o BIM tem o potencial para revolucionar a construção civil, alinhando-a, em termos de uso de tecnologia de informação, com outras indústrias.

Figura 1 - Metodologia BIM x Método tradicional

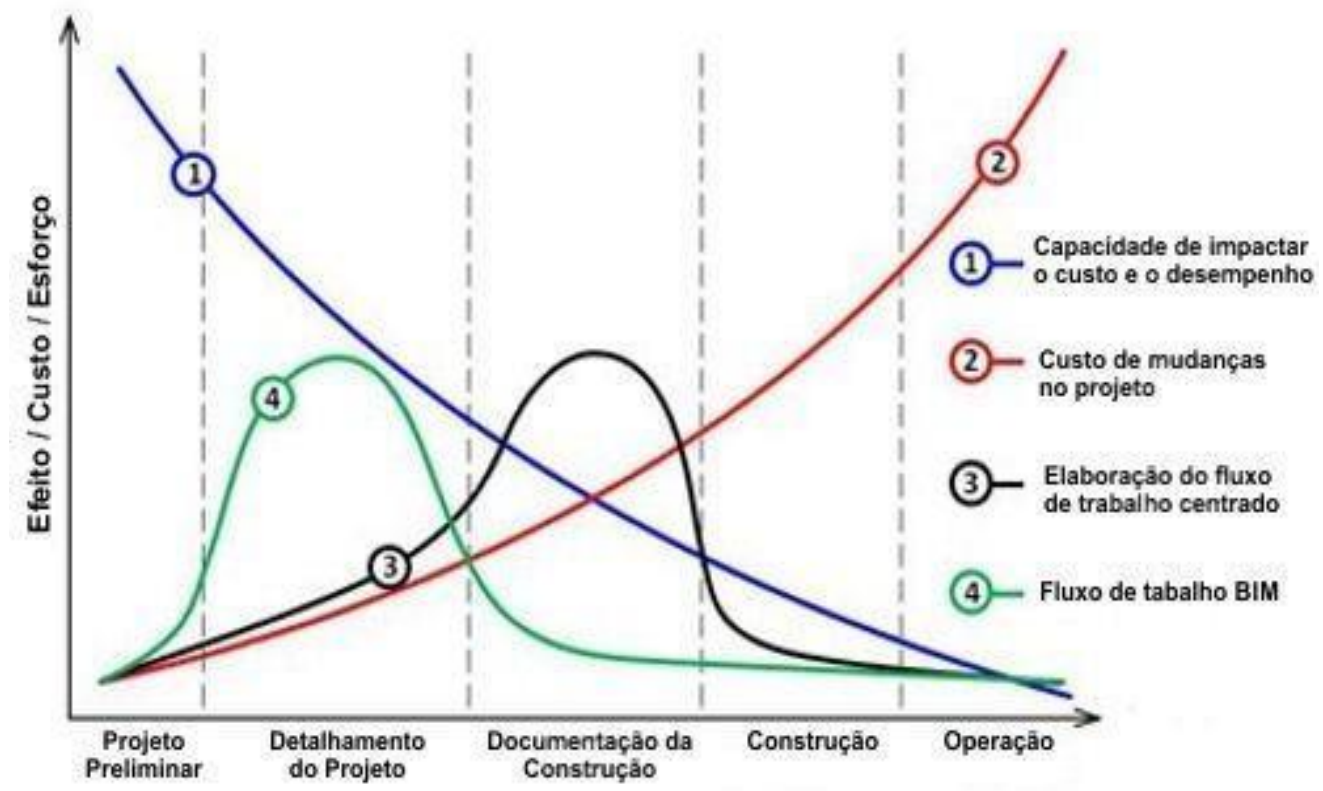

Fonte: https://www.blogdaarquitetura.com/saiba-como-o-bim-ajuda-no-desempenho-de-uma-edificacao/

A Figura 1 apresenta um comparativo entre o uso do BIM e do método tradicional no decorrer de um projeto. Utilizando a tecnologia de Modelagem da Informação da Construção é muito mais provável prever erros e corrigi-los antes da execução da obra, etapa em que o custo das alterações do projeto crescem em uma taxa muito mais alta se comparada a fase de anteprojeto.

Sabendo da relevância desta metodologia, no Brasil o decreto ${ }^{\circ} 10.306$ de 2 de abril de 2020 regulamenta a Estratégia Nacional de Disseminação do Building Information Modelling e prevê a utilização do BIM em obras e serviços de engenharia realizados por órgãos da administração pública federal.

A Estratégia BIM BR lançada em 2018, tem como foco promover um ambiente adequado ao investimento em BIM e a sua difusão no país, tendo como um dos objetivos estimular a capacitação em BIM, estimulando sua maior inserção nas disciplinas de graduação e pósgraduação em Engenharia e Arquitetura.

No cenário internacional, a adoção de experiências didáticas relacionadas ao BIM nas universidades tem ocorrido de forma muito mais madura, sendo este abordado ao longo de vários momentos da formação do profissional, pela grande demanda de mercado existente em outros países (BANDEIRA, 2019). Para a autora, as universidades nacionais estão ainda buscando traçar estratégias de ensino, a medida que a tecnologia se difunde. 
No centro de tecnologia da Universidade Federal, as primeiras ações com o intuito de disseminar o BIM no ambiente acadêmico iniciaram com eventos pontuais, por meio de palestras e posteriormente cursos, introduções pontuais em disciplinas de desenho para engenharia, bem como o desenvolvimento de pesquisas relacionadas.

Em 2015, surgiu um grupo de estudos voltado para o aprendizado da metodologia BIM formado por alunos que sentiram a necessidade de desenvolver esse conhecimento dentro da universidade. Em 2017, o grupo de estudos foi oficializado como projeto de extensão vinculado ao Departamento de Integração Acadêmica e Tecnológica da Universidade Federal do Ceará. Assim surge o Escritório de Projetos Integrados de Engenharia - EPE, um projeto de extensão que tem a missão de fomentar a filosofia no meio acadêmico, simultaneamente proporcionando uma vivência de gestão e a experiência de participar de um escritório de projetos a seus membros.

No mesmo ano, foi realizada uma pesquisa pelo Centro Acadêmico do Curso de Engenharia Civil do Campus de Fortaleza com o fito de analisar a demanda de alunos por uma disciplina voltada especificamente para o ensino da metodologia BIM. A pesquisa também revelou a relação dos estudantes e o grau de contato deles com a filosofia na época. Os resultados obtidos através do questionário foram o ponto de partida para a criação da disciplina de Modelagem da Informação e da Construção (MIC), a primeira disciplina completamente voltada para o ensino de BIM no curso de engenharia civil.

No tocante a eventos que tem o propósito de fomentar o BIM no meio acadêmico, tem-se em 2016 a realização do $1^{\circ}$ Workshop de BIM, evento realizado pelo projeto de extensão Escritório de Projetos Integrados de Engenharia e que ocorrerá em sua $4^{\mathrm{a}}$ edição esse ano. $\mathrm{O}$ workshop tem a proposta de promover a troca de experiências entre academia e mercado de trabalho, contando com a participação de grandes expoentes do mercado de engenharias e empresas nacionais e internacionais na realização de palestras, oficinas e minicursos. Ainda sob o enfoque de evento, a cada dois anos, em anos pares, ocorre o Seminário Internacional de Construção Enxuta - CONENX, que em suas últimas edições incorporou a temática BIM no evento.

Em 2019, o Encontro Nacional sobre o Ensino de BIM aconteceu em Fortaleza na Universidade Federal do Ceará, contando com a participação de estudantes de graduação e pósgraduação, docentes e profissionais do mercado e promovendo debates relevantes acerca do ensino de BIM pelo Brasil, sobretudo experiências didáticas.

\section{OBJETIVOS}

Comparar a percepção sobre o avanço da metodologia BIM no curso de Engenharia Civil do Centro de Tecnologia da UFC entre os anos de 2017 e 2019, pontuando ações voltadas para a inserção de BIM na universidade nesse período.

\section{METODOLOGIA}

Inicialmente, foi realizada a revisão bibliográfica de trabalhos relacionados ao tema BIM na Universidade Federal do Ceará ao longo desses três anos, dentre monografias, dissertações de mestrado e artigos científicos. Além disso, fez-se um levantamento de eventos dentre congressos e palestras organizados com a participação direta da universidade voltadas para esta tema, bem como de cursos ou minicursos realizados na UFC neste período. Dessa forma, serão analisadas por meio de indicadores quantitativos ações de inserção do BIM na universidade 
divididas nas categorias: produção científica, eventos e capacitações, de modo que os resultados serão analisados sob o olhar dicotômico do mercado de trabalho e da academia.

Concomitantemente, foi aplicada uma pesquisa, adotando como parâmetros estatísticos um grau de confiança de $95 \%$ e uma margem de erro de $10 \%$ para assegurar a confiabilidade da amostra, por meio de um formulário eletrônico buscando um diagnóstico da situação atual do nível de conhecimento de BIM dos estudantes do curso de engenharia civil do Campus do Pici - Fortaleza, além de obter um panorama do ensino de BIM no curso, em quais disciplinas os discentes têm contato com a metodologia e se há projetos de extensão e outras organizações estudantis que procuram levar esse conhecimento aos alunos. Essa pesquisa será analisada de modo comparativo com a pesquisa similar aplicada pelo Centro Acadêmico de Engenharia Civil do Campus de Fortaleza em 2017. Em ambas as pesquisas foi questionado aos estudantes se tiveram algum contato com BIM no decorrer da graduação e qual o grau de importância de aprofundar essa metodologia na graduação na visão deles.

\section{RESULTADOS}

\subsection{Levantamento de Dados}

Realizando a análise quantitativa das iniciativas em suas respectivas categorias, tem -se na figura 2 o resultado do levantamento da categoria de produção científica no período analisado.

Figura 2 - Levantamento da produção científica publicada entre 2017 e 2019

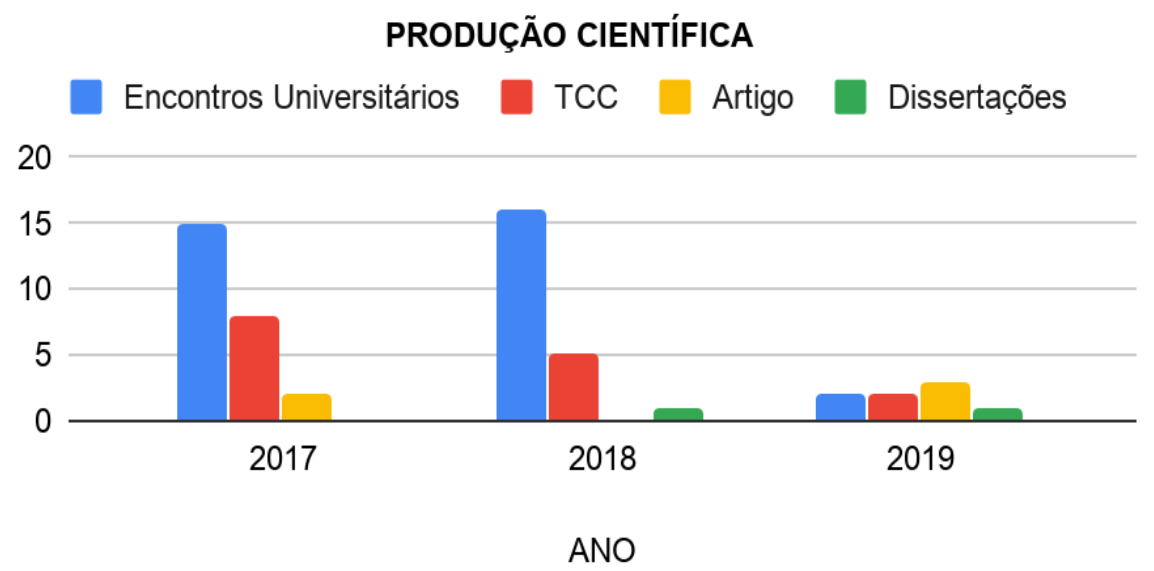

Fonte: Autoral.

Em 2017, tem-se trabalhos de conclusão de curso de alunos do curso de Engenharia Civil sobre esse assunto, de modo que 2017 foi o ano com maior número de TCCs relacionados a BIM. Segundo Gabriel Medeiros, um dos estudantes que publicou um TCC relacionado a BIM em 2017, diversos alunos obtiveram contato com a metodologia BIM no Exterior por meio do programa Ciências Sem Fronteiras, e ao retornar para o Brasil, desenvolveram seus Trabalhos de Conclusão de Curso relacionados a área. Nos anos posteriores, percebeu-se ainda desenvolvimento de trabalhos relacionados a metodologia, porém em menores números. Dentre os trabalhos, foram desenvolvidos temas relacionados a automação de projetos em BIM, Ensino de BIM por meio de metodologias ativas, dentre outros trabalhos de bastante relevância para o meio tecnológico. Ainda, foram realizados levantamentos de dissertações neste mesmo período, as quais os temas constam na Tabela 2. Para 2020, tem-se duas dissertações previstas. É possível 
verificar os trabalhos desenvolvidos no repositório institucional da Universidade Federal do Ceará em: http://www.repositorio.ufc.br/.

Em contrapartida, observa-se pela figura 3 uma constância nos números de eventos (categoria que abrange desde congressos a palestras avulsas) e um aumento ao longo dos anos no número de capacitações com temas relacionados a metodologia BIM promovidas no ambiente universitário, dentre cursos e minicursos (Figura 4). Muitas dessas capacitações ofertadas foram promovidas pelos próprios discentes representados por organizações estudantis como o Centro Acadêmico, Programa de Educação Tutorial (PET) e Escritório de Projetos Integrados em Engenharia (EPE).

Figura 3: Número de eventos nos anos 2017, 2018 e 2019.

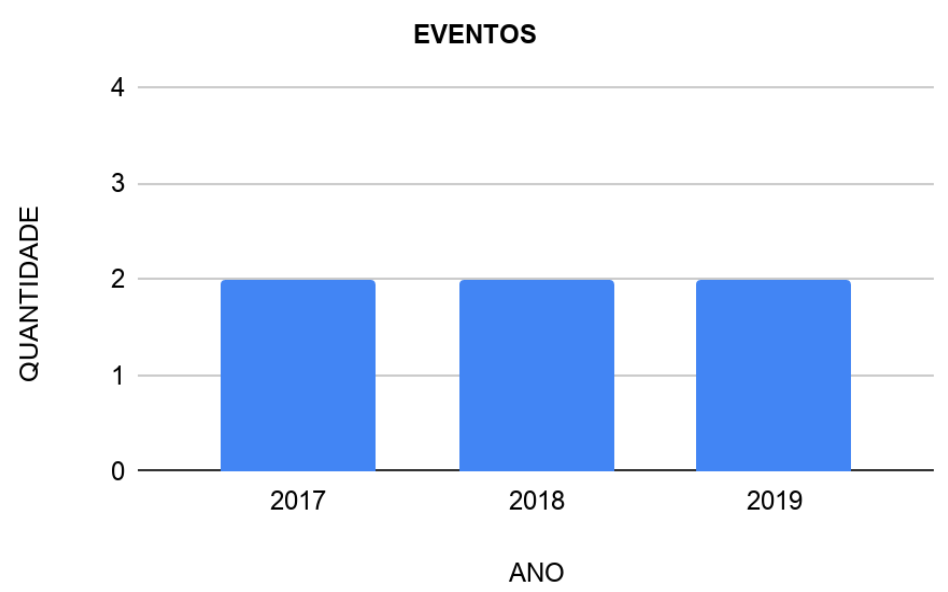

Fonte: Autoral.

Figura 4: Número de capacitações nos anos 2017, 2018 e 2019.

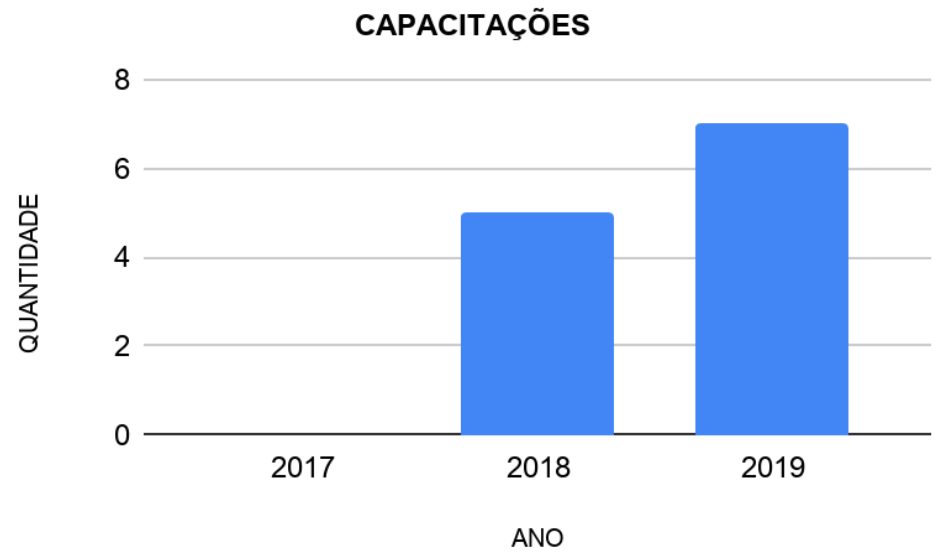

Fonte: Autoral

\subsection{Diagnóstico: BIM no curso de Engenharia Civil.}

As Figuras 5 e 6 mostram respectivamente os resultados das pesquisas de 2017 e 2020 quando os alunos foram questionados sobre o contato com a metodologia BIM em disciplinas da graduação. É perceptível que ao longo desses 3 anos o conceito de BIM está sendo mais difundido no curso, no entanto, de forma ainda muito superficial ou pontual. 
Figura 5: Respostas de 2017.

Você já teve contato com a tecnologia BIM na graduação?

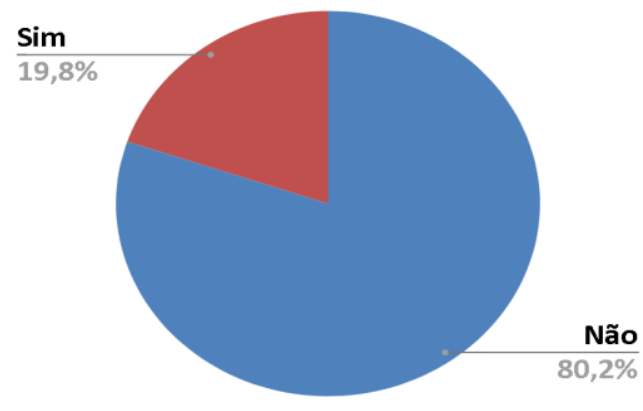

Fonte: Autoral

Figura 6: Respostas de 2020

Você já teve contato com BIM em alguma disciplina do seu curso?

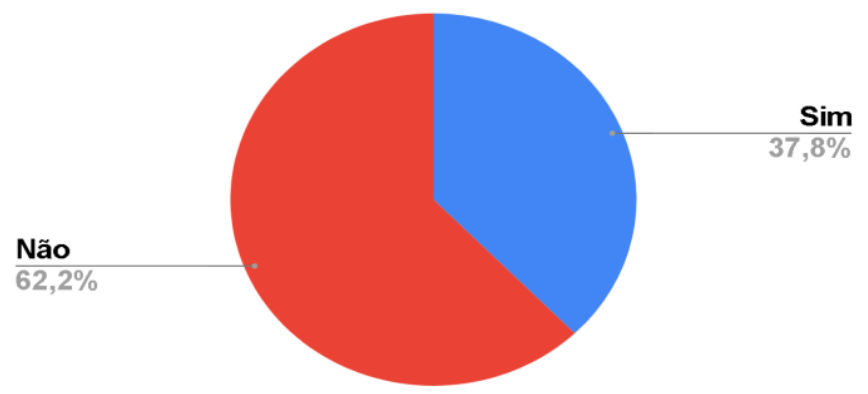

Fonte: Autoral

Quando questionados acerca do grau de importância, em uma escala de 1 a 5, que eles dão ao aprofundamento desta metodologia no curso têm-se as figuras 7 e 8 revelando que a percepção da importância do aprofundamento no BIM na graduação também evoluiu entre os discentes.

Figura 7: Pesquisa de 2017

Que nível de importância você dá a um estudo mais aprofundado da tecnologia BIM na graduação?

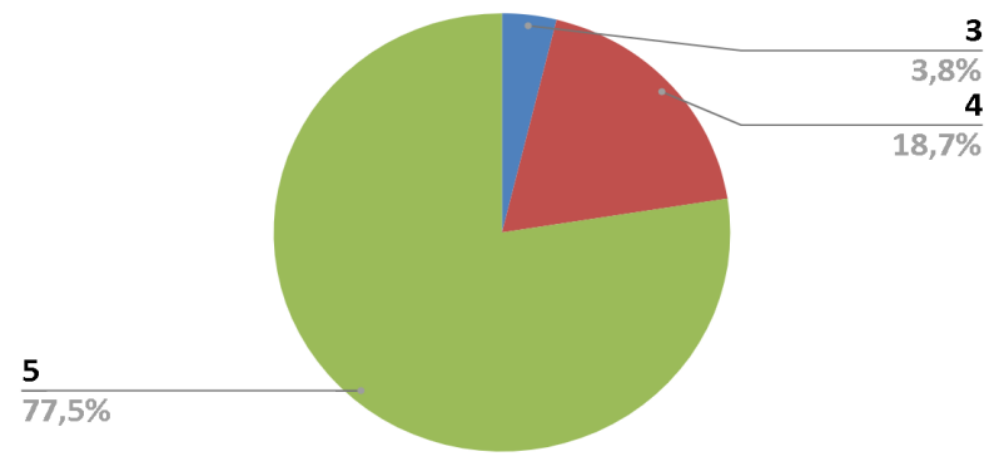

Fonte: Autoral 
Que nível de importância você dá a um estudo mais aprofundado da tecnologia BIM na graduação?

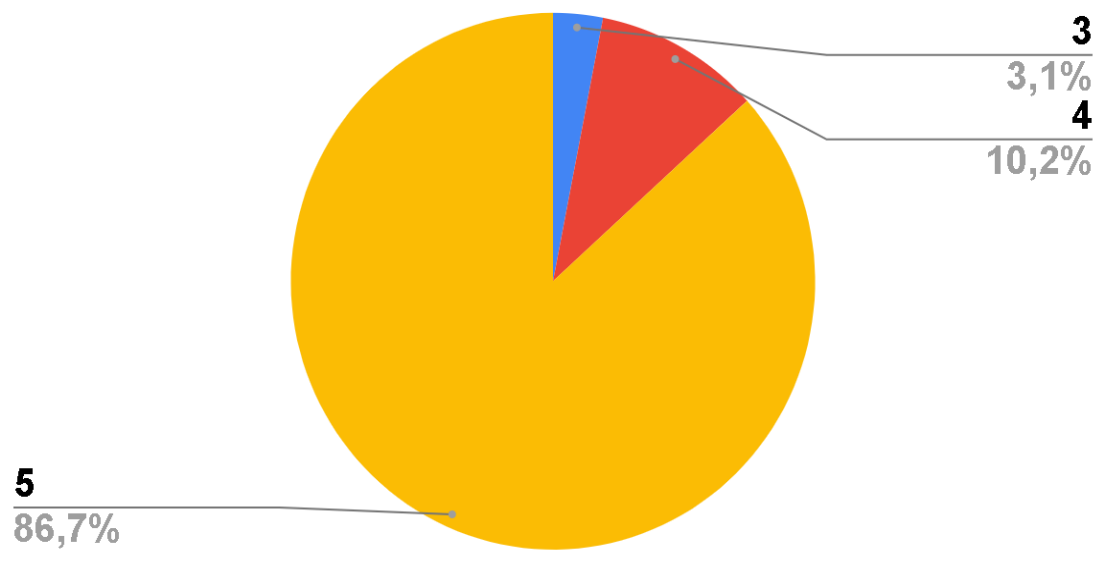

Fonte: Autoral

Acerca da pesquisa mais recente, $97 \%$ dos alunos do curso de engenharia civil responderam que conhecem a metodologia BIM, ainda que esse conhecimento se revele em diferentes níveis de percepção. Quando questionados sobre as iniciativas de BIM na UFC, aproximadamente $47 \%$ responderam que conheciam (Figura 9) e 65\% destes citaram o Escritório de Projetos Integrados de Engenharia como esta iniciativa.

Figura 9:Percepção acerca das iniciativas de BIM na UFC
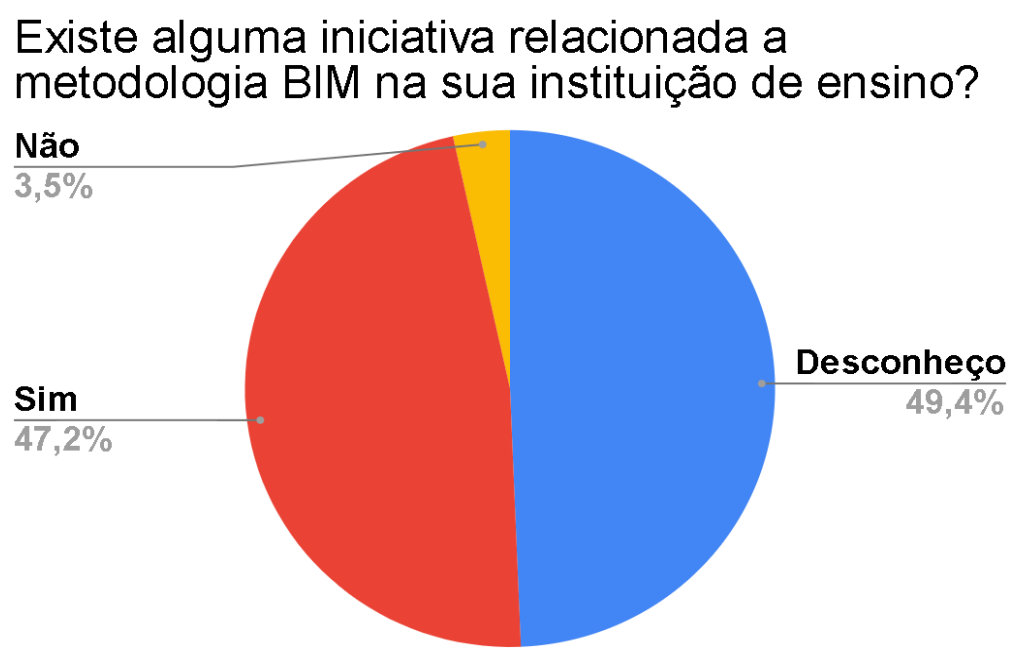

Fonte: Autoral.

Quando questionados sobre quais disciplinas do curso abordam a metodologia, os estudantes citaram Modelagem da Informação e da Construção (disciplina optativa totalmente focada em BIM que surgiu a partir da pesquisa de 2017), bem como disciplinas com o uso pontual de ferramentas BIM (Autodesk Revit e Civil 3D) dentre as quais Gerenciamento para Construção Civil, Projeto e Construção de Edifícios e Projeto e Construção da Infraestrutura Viária. 


\section{CONSIDERAÇÕES FINAIS}

Diante do exposto, no ano de 2017, o grande número de discentes que retornaram do exterior para a UFC devido ao programa ciência sem fronteiras, trouxeram conhecimentos adquiridos durante o intercâmbio, uma vez que nas universidades estrangeiras o uso de BIM já estava consolidado por meio de disciplinas, contribuindo inclusive para o embasamento da necessidade da disciplina de Modelagem da Informação e da Construção - MIC, no contexto das engenharias. Além disso, neste mesmo ano, a UFC esteve envolvida na realização de eventos brasileiros como SBTIC e SIBRAGEC, além de iniciativas internas de fomento a disseminação da metodologia BIM. Em 2018, foi iniciada a primeira turma de MIC, disciplina optativa para o curso de engenharia civil. Os alunos da turma produziram trabalhos de conclusão de curso relacionados a área de BIM, trazendo temáticas como avaliações pedagógicas de ensino aprendizagem e geometria de processos, temas abordados pela disciplina. Assim, é perceptível a influência das iniciativas relacionadas a BIM na vida acadêmica dos discentes. Tangente a isso, foram realizados eventos internos como o $3^{\circ}$ Workshop de BIM, com atrações regionais, nacionais e internacionais, e ainda, o Seminário Internacional Sobre Construção Enxuta com o tema: Inovação na Gestão da Construção: O uso de lean, BIM e sua integração. Em 2019, notou-se uma diminuição do número de TCCs em relação ao ano anterior, por outro lado o número de artigos aumentou e o número de dissertações de mestrado se manteve. Em relação ao número de eventos realizados, também foi mantido. $\mathrm{O}$ que nos leva a concluir que os discentes em sua grande parte estão buscando preferencialmente o desenvolvimento referente a capacitações relacionadas à metodologia para atuação no mercado, não focando em produções científicas relacionadas ao tema. As pesquisa mostram que, apesar de o conceito de BIM estar melhor difundido bem como a percepção de sua relevância, o BIM não se encontra consolidado de forma integrada e consistente no curso, tendo-se aplicações pontuais de ferramentas BIM em algumas disciplinas.

A partir das análises é notório a importância de projetos extracurriculares e organizações estudantis na disseminação desse conhecimento e no preparo dos estudantes para o mercado de trabalho, dentre eles temos o EPE, que planejou ações em diversas disciplinas do curso de Engenharia Civil para o ano de 2020, mas devido a crise mundial do Covid-19, as ações para disseminação de BIM dentro da universidade foram reformuladas. O EPE organizou palestras online, pela plataforma Instagram, além de oferecer suporte às disciplinas de Desenho para Engenharia e Gerenciamento para Construção Civil, com o auxílio no uso das ferramentas no desenvolvimento de trabalhos e ainda, participando das aulas online.

\section{Agradecimentos}

Gostaríamos de agradecer ao Departamento de Integração Acadêmica e Tecnológica (DIATEC) do Centro de tecnologia da Universidade Federal do Ceará e ao Projeto de extensão Escritório de Projetos Integrados de Engenharia (EPE) vinculado ao departamento, que contribui continuamente com o fomento da metodologia BIM no meio acadêmico e tecnológico.

\section{REFERÊNCIAS}

ADDOR, M. R. A. Proposta de Metodologia de Avaliação para Salas de Coordenação de Projetos em BIM. 2015. 176 f. Dissertação (Mestrado) - Escola Politécnica, Universidade de São Paulo, São Paulo, 2015. 
BANDEIRA, L. R. Tendências atuais para a inserção do BIM no ensino superior. Revista Científica Educandi \& Civitas, Tocantins, v.2, n.2, 2019.

BRASIL. Presidência da República. DECRETO No 10.306, DE 2 DE ABRIL DE 2020. Disponível em: http://www.in.gov.br/web/dou/-/decreto-n-10.306-de-2-de-abril-de-2020-2510 68946. Acesso em: 31 mai. 2020.

EASTMAN,Chuck et al. Manual BIM: Um guia de modelagem da informação da construção para arquitetos, engenheiros, gerentes, construtores e incorporadores. $2^{\text {a }}$ edição. Porto Alegre: Bookman, 2014.

KYMMELL, Willem. Building Information Modeling - Planning and Managing Construction Projects with 4D CDA and Simulations. Ebook. McGraw-Hill, 2008.

UNIVERSIDADE FEDERAL DO CEARÁ. Repositório Institucional. Disponível em: http://www.repositorio.ufc.br/. Acesso em: 02 mai. 2020

VAN NEDERVEEN, G. A.; TOLMAN, F. P. Modeling multiple views on buildings. Automation in Construction, Amsterdam NL, v. 1, issue 3, p. 215-224, Dec. 1992.

\title{
DIAGNOSIS OF METHODOLOGY BIM BETWEEN THE YEARS 2017 AND 2019 IN TECHNOLOGY CENTER UFC.
}

\begin{abstract}
:
The BIM methodology is gaining more and more importance in Brazil and the BIM BR strategy corroborates this. In this sense, we aim to carry out a diagnosis on the perception of the BIM methodology in the context of the Federal University of Ceará, between the years 2017 and 2019. For this purpose, a survey was carried out in 2017 to students at the UFC technology center, where they were questioned about the perception of BIM philosophy in the course, its relevance and interest in the discipline focused on BIM. In 2020, a new research was carried out with the students for comparative purposes of the data. Simultaneously, actions related to BIM in the university carried out in this period were classified in the categories: scientific production, events and training was carried out. Among the categories, it was possible to notice that in 2017 there was the largest number of undergraduate thesis that dealt with the methodology, a fact that coincided with the return of several students from abroad. On the other hand, the number of related events organized at the university has been increasing, demonstrating the relevance on the theme, among them we have national and international events. Furthermore, the number of training courses offered at the university has also shown to be increasing, which corroborates the importance of the methodology. Therefore, in a scenario where we have the BIM BR Strategy as a strong incentive for the use of the methodology, we can infer that although the scientific production related to the theme has decreased, the other growing categories show that the greatest concern among students is the preparation to ingress in the market.
\end{abstract}

Keywords: Diagnosis. BIM. UFC. 